\title{
Response to treatment with imatinib mesylate in previously treated chronic-phase chronic myeloid leukemia patients in a hospital in Brazil
}

\author{
C.A.P. Silveira ${ }^{1}$, M.B. Daldegan ${ }^{1}$ and I. Ferrari ${ }^{2}$
}

${ }^{1}$ Núcleo de Hematologia e Hemoterapia, Hospital de Base do Distrito Federal, Secretaria de Estado da Saúde do Distrito Federal, Brasília, DF, Brasil ${ }^{2}$ Departamento de Genética e Morfologia, Instituto de Ciências Biológicas, Universidade de Brasília, Brasília, DF, Brasil

Corresponding author: C.A.P. Silveira

E-mail: carlosapsilveira@gmail.com

Genet. Mol. Res. 10 (3): 2038-2048 (2011)

Received September 21, 2010

Accepted February 8, 2011

Published September 15, 2011

DOI http://dx.doi.org/10.4238/vol10-3gmr1073

\begin{abstract}
We analyzed the results of treatment with imatinib mesylate in 70 patients with chronic-phase chronic myeloid leukemia who had previously been treated (with second-line or higher imatinib), many of them in a late chronic phase. The median followup period was 60.5 months (range 3-100 months). Our objective was to assess the efficacy and safety of treatment. The mean dose was $400 \mathrm{mg}$ per day. The hematologic response rate was $92.1 \%$ at six months, while the cumulative rates of major and complete cytogenetic responses were 73.6 and $66.3 \%$, respectively. Molecular response rate improved slowly and steadily over time, reaching $65.8 \%$ at 60 months, remaining stable for up to 96 months. The five-year progression-free survival and overall survival were 84 and $89 \%$, respectively. Cytogenetic response by 12 months significantly correlated with overall survival $(\mathrm{P}=0.0007)$ and progression-free survival $(\mathrm{P}=0.0280)$. Sokal risk score did not differ significantly between subgroups. The medication was well tolerated, with only
\end{abstract}


$16 \%$ of patients showing hematologic toxicity grades 3 and 4 . At the end of the assessment, $57 \%$ of the patients were still on imatinib mesylate; most of those who discontinued treatment (17/30) did so because of unsatisfactory response. Treatment with imatinib mesylate in previously treated chronic-phase chronic myeloid leukemia induced durable responses in a high proportion of patients and was related to satisfactory long-term and event-free survival.

Key words: Chronic myeloid leukemia; Imatinib mesylate; Cytogenetic response

\section{INTRODUCTION}

Chronic myeloid leukemia (CML) is a chronic myeloproliferative disorder characterized by the presence of Philadelphia chromosome (Ph-positive CML), which is the result of a balanced translocation between chromosomes 9 and 22 (Sawyers, 1999; Vardiman et al., 2008). The molecular consequence of this translocation is the resulting hybrid BCR-ABL1 gene, which encodes the chimeric protein p210bcr-abl1 with constitutively and deregulated tyrosine kinase activity (Quintás-Cardama and Cortes, 2009).

The oncogenic potential of the BCR-ABL1 hybrid protein has been proven by its ability to transform hematopoietic progenitor cells in vitro and in vivo (Sawyers, 1999; Thijsen et al., 1999). CML occurs in three phases (chronic, accelerated and blast crisis) and is usually diagnosed during chronic phase (Bortolheiro and Chiattone, 2008).

The mechanism by which the $\mathrm{p} 210 \mathrm{bcr}-\mathrm{ab} 11$ protein promotes the transition from a benign state to a malignant state is not completely understood. However, the juxtaposition of sequences encoded by the BCR gene to the ABL1 gene results in critical functional changes that lead to increased cell proliferation, affect cellular differentiation and block apoptosis (Goldman and Melo, 2003).

Before imatinib mesylate (IM), the treatment of Ph-positive CML included hydroxyurea, interferon alpha (INF- $\alpha$ ), and allogeneic hematopoietic stem cell transplantation (Silver et al., 1999). The advent of imatinib, which targets the tyrosine kinase activity of oncogenic proteins encoded by BCR-ABL1 (Druker et al., 1996), quickly and dramatically changed the treatment of CML (Druker et al., 2001) and led to major changes in its management (Baccarani et al., 2006). The most significant of these changes occurred after publication of the IRIS study (International Randomized Study of Interferon and STI571), which showed the clear superiority of imatinib compared to INF- $\alpha$ and cytarabine at low doses as initial treatment for CML (O'Brien et al., 2003). Currently, imatinib mesylate is considered to be the standard treatment for newly diagnosed CML (Baccarani et al., 2009). Nevertheless, the interest in previously treated chronic phase patients is still substantial, because IM may not be fully available as front-line treatment worldwide for economic reasons (Palandri et al., 2008).

Since March 2002, IM has been used in the hematology service at the Hospital de Base do Distrito Federal (Brasilia, Brazil). This article describes the results of second-line IM therapy for chronic-phase Ph-positive CML patients at this hospital, since there are no previous data on this population of patients. The main goal was to evaluate the efficacy and survival rate with the drug. 


\section{PATIENTS AND METHODS}

\section{Inclusion criteria}

Patients were eligible for the study if they were 18 years of age or older and had Phpositive chronic phase CML and received IM as second-line or higher option of treatment in the period between March 2002 and January 2009. We included both patients previously monitored by the service and those who were admitted as of March 2002, if previously treated and naive to imatinib. The diagnosis of CML was suspected by clinical and hematological criteria and was established by conventional karyotype analysis, FISH (fluorescence in situ hybridization) or reverse transcriptase polymerase chain reaction (RT-PCR) for BCR-ABL. Chronic phase was defined by the presence of less than $15 \%$ blasts, less than $20 \%$ basophils, and less than $30 \%$ blasts and promyelocytes in peripheral blood and bone marrow, and platelet count exceeding 100,000/ $\mathrm{mm}^{3}$ (Kantarjian et al., 2002a).

\section{Exclusion criteria}

The study excluded patients who were exclusively on IM or used it after hydroxyurea only, pregnant women, and patients diagnosed with CML admitted to the service and who had never taken imatinib because they were referred for other forms of treatment (allogeneic hematopoietic stem cell transplantation).

\section{Treatment}

Patients were treated with IM at $400 \mathrm{mg}$ daily, orally and continuously with dose adjustments, either for toxicity (with decreasing the dose) or for unsatisfactory response (raising the dose to 600 or $800 \mathrm{mg}$ daily), in accordance with guidelines provided in the literature so far valid (Baccarani et al., 2006; Goldman, 2007).

\section{Study design and statistical analysis}

This was a descriptive study based on institutional data. The aim was to evaluate the efficacy and safety of IM treatment in our institution. Patients were analyzed for overall survival, event-free survival, progression to accelerated-phase CML or blast crisis, and hematologic, cytogenetic and molecular responses. Hematologic response was defined as previously described (Kantarjian et al., 2002a). The cytogenetic response was defined according to the number of $\mathrm{Ph}$ chromosome-positive cells in metaphases and was categorized as complete (zero percent $\mathrm{Ph}$ chromosome-positive cells in metaphase in bone marrow), partial (1-35\%), minor (36-65\%), minimal (66-95\%), and no response (>95\%) (Kantarjian et al., 2002a). Response was defined as major if the patient had a complete or partial response. Molecular response was described as previously defined (Kantarjian et al., 2002a; Hughes et al., 2003; Funke et al., 2005). Occurrence of events in patient outcome was defined as: death (any cause), progression to advanced stages of disease (accelerated phase and/or blast crisis) (Cortes and Kantarjian, 2003; Giles et al., 2004) and loss of responses (hematologic, cytogenetic or molecular). Eventfree survival was defined from the time of start of treatment to the first documentation of one 
of the above-mentioned events, and it was censored at the time of treatment discontinuation or the last visit, whichever came first. Progression-free survival was defined as the time between start of therapy and the onset of an accelerated phase or blast crisis. Patients without progression were censored at the time of treatment discontinuation or at the time of the last visit. Overall survival was determined as the time from treatment initiation to death from any cause and was censored at the time of treatment discontinuation or on the date of the last visit. Hematologic failure was considered if patients failed to achieve complete hematologic response at six months or if they lost their hematologic remission at any time. Cytogenetic failure was considered if patients failed to achieve major cytogenetic response (MCR) at 12 months and complete cytogenetic response (CCR) at 18 months, as well as loss of MCR at any time. Overall survival, progression-free survival and event-free survival were all evaluated using the Kaplan-Meyer methods, with statistical significance (P value) assessed using the log-rank test.

\section{Assessment of patients}

Monitoring was performed according to the routine of the Hematology Department of Hospital de Base do Distrito Federal. In general, hematologic tests were done weekly, until stabilization, monthly during the first six months and every three months thereafter. Cytogenetic tests were scheduled for six, twelve and eighteen months of treatment and then annually or at any time according to discretion of the treating physician. Molecular tests by RT-PCR was scheduled every three months, from the eighteenth month of treatment or after obtaining a complete and confirmed cytogenetic response.

\section{Ethical aspects}

The study was approved by the Institutional Research Ethics Committee of the Secretaria de Estado de Saúde do Governo do Distrito Federal.

\section{RESULTS}

Seventy patients were eligible for the study. The median time between diagnosis and initiation of treatment was 16 months (range: 0-208). Overall, INF- $\alpha$-based regimen was the principal first-line option of treatment. Two patients exhibited non-hematologic intolerance grades 3 and 4 to interferon and did not complete a month of use of this drug. Forty (57.1\%) patients were on imatinib for more than 12 months after diagnosis (late chronic phase). The median follow-up was 60.5 months (range 3-100 months). The main clinical and hematologic features at diagnosis of the patients are summarized in Table 1.

\section{Efficacy of imatinib}

After six months, complete hematologic response was achieved in $92.1 \%$, and it was achieved in $94.4 \%$ at some point during the study. The results of cytogenetic analysis at six months were available in $39(55.7 \%)$ patients. The number of patients with MCR, CCR, partial cytogenetic response, and minor cytogenetic response were 26 (66.7\%), 12 (30.8\%), 14 (35.9\%), and 9 (23.1\%), respectively. The cumulative rates of MCR and CCR were 73.6 and 


\section{Table 1. Patients' characteristics at diagnosis.}

\begin{tabular}{ll}
\hline Variables & \\
\hline Median age, years (range) & $44(19-73)$ \\
Age $\geq 60$ years & $11(15.7)$ \\
Gender (male/female) & $34(48.6) / 36(51.4)$ \\
Spleen size (cm) & $43(61.4)$ \\
Available data & $15(21.4)$ \\
Spleen $\geq 10 \mathrm{~cm}$ bcm & $35(50.0)$ \\
Sokal risk group & $16(45.7)$ \\
Available & $7(20.0)$ \\
Low risk & $12(34.3)$ \\
Intermediate risk & $12.2(6.2-15.7)$ \\
High risk & $115,600(6300-651,000)$ \\
Hematologic variable: median (range) & $473,000(126,000-1,750,000)$ \\
Hemoglobin level $(\mathrm{g} / \mathrm{dL})$ & $0(0-11)$ \\
White cell count $\left(\mathrm{cells} / \mathrm{mm}^{3}\right)$ & $0(0-12)$ \\
Platelet count (cells/mm $)$ & \\
Blasts in peripheral blood & \\
Basophils in peripheral blood &
\end{tabular}

$66.3 \%$, respectively. They are shown in Figure 1 and indicate a trend toward improvement over time both in MCR and CCR with stabilization of MCR rate from the 30th month on and CCR rate from the 40th month on. Seven of fifty-six patients with available data (12.5\%) lost their cytogenetic response throughout the observation period. The median time to loss of cytogenetic response was 23 months (range: 12-60 months).

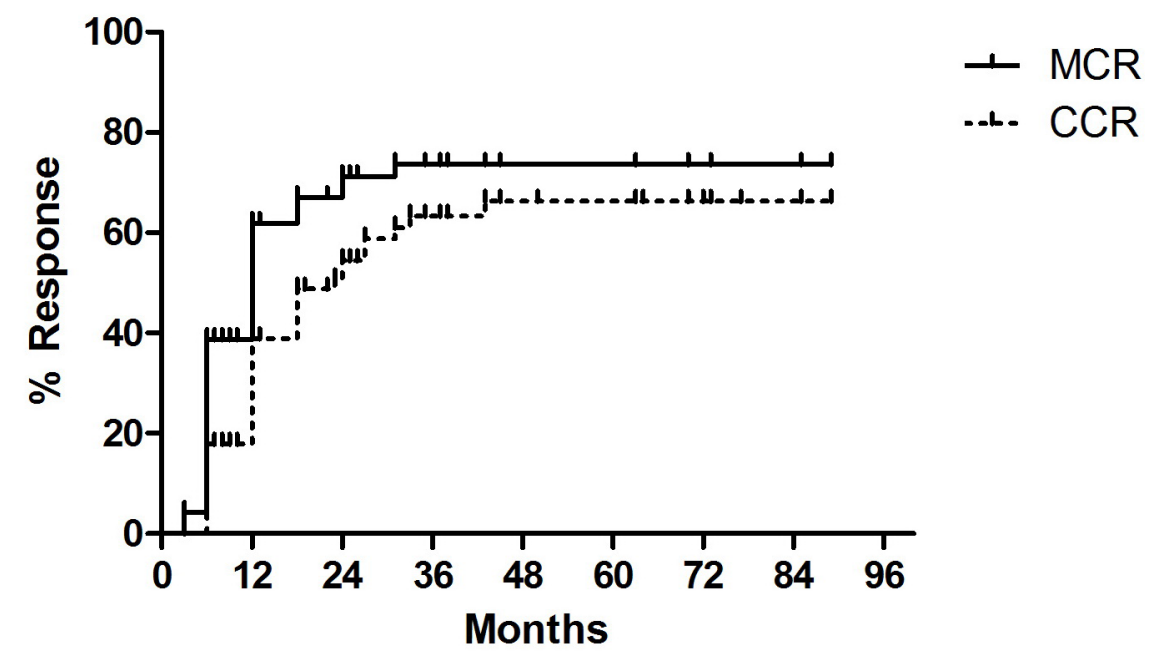

Figure 1. Time to cytogenetic responses: major (MCR) and complete (CCR).

The analysis of molecular response was performed in patients who achieved a CCR. The cumulative rate of major molecular response is shown in Figure 2. There was a tendency toward progressive improvement until the 60th month. The estimated 5-year cumulative rate of MCR was $65.8 \%$. Only one patient (2.9\%) showed loss of molecular response at the end of observation. 


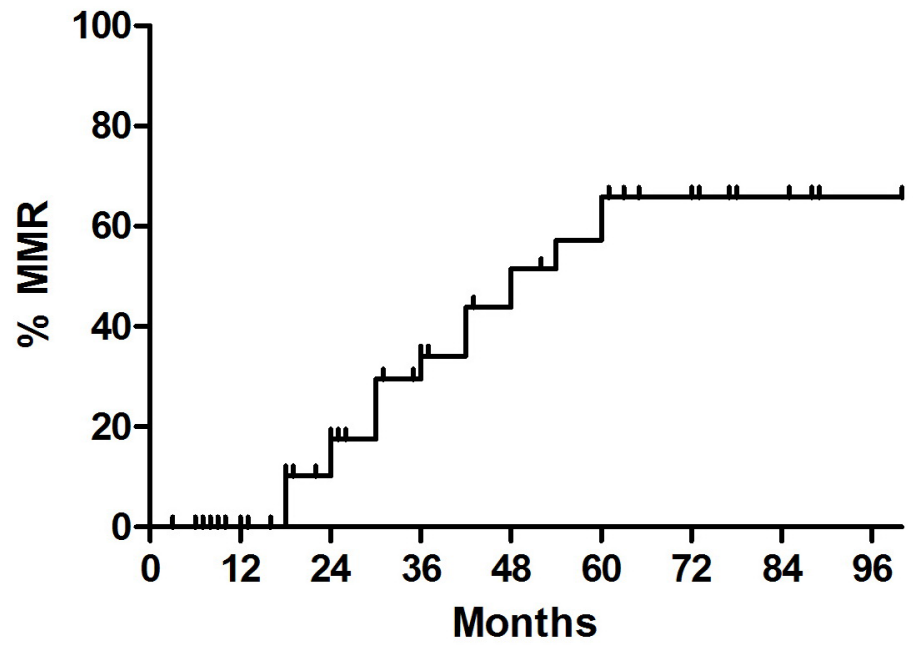

Figure 2. Cumulative rate of major molecular response (MMR).

Sokal score (Sokal et al., 1984) was not associated with achievement of cytogenetic response $(\mathrm{P}=0.4898)$ (Figure 3$)$.

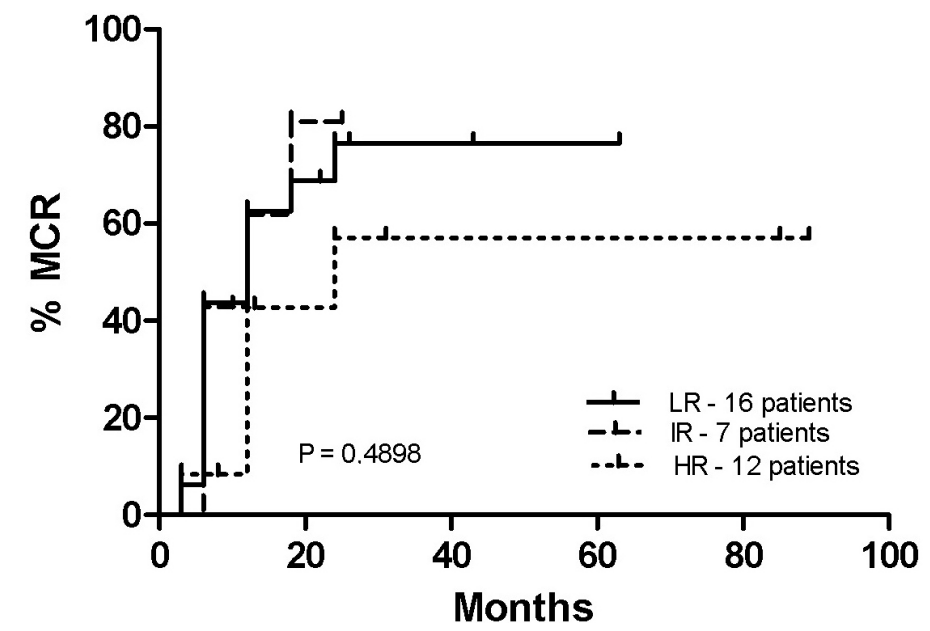

Figure 3. Sokal risk score and probability of achieving major cytogenetic response (MCR). LR = low risk; IR = intermediate risk; $\mathrm{HR}=$ high risk.

\section{Long-term outcomes}

The estimated progression-free survival (PFS) rate at 60 months was $84.2 \%$ (Figure 4). Sixty-four (91.4\%) among the 70 patients were alive at the end of the observation period. There were six deaths: five related to progression of the disease and one related to sepsis after skin infection. Overall survival rate was $89.1 \%$ (Figure 5). 


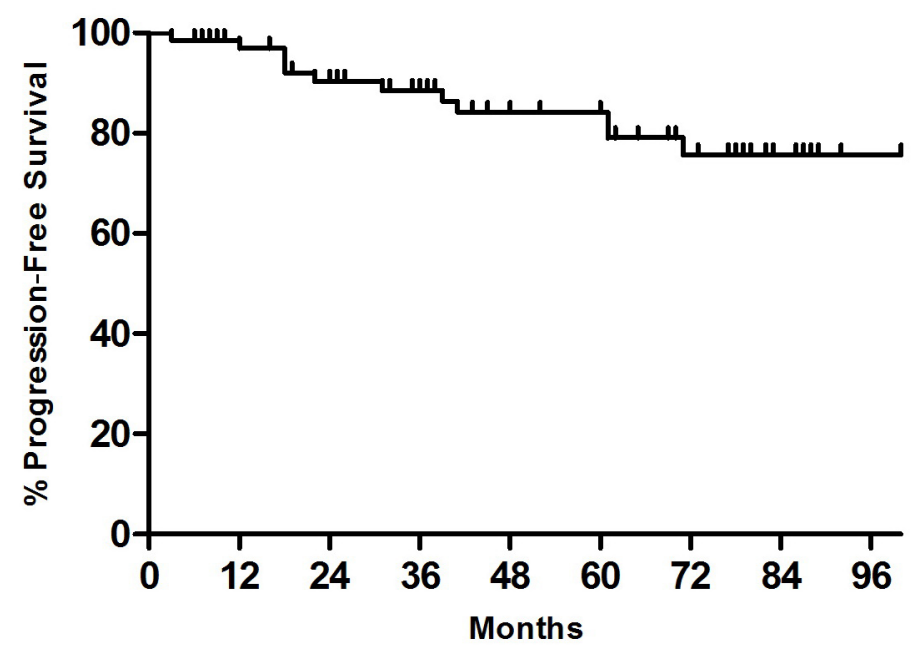

Figure 4. Progression-free survival.

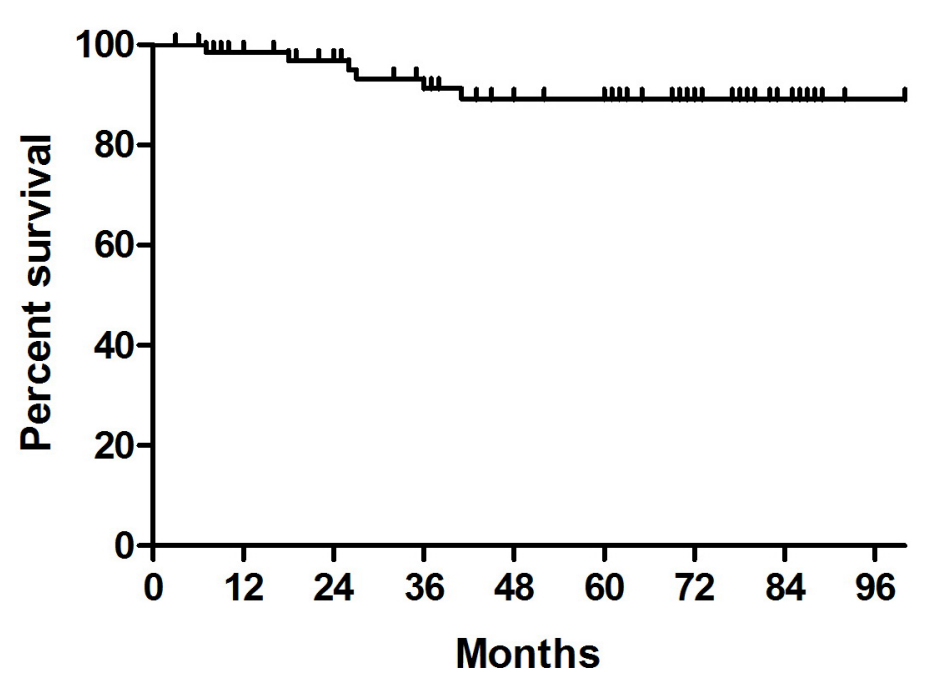

Figure 5. Overall survival.

Achievement of cytogenetic response by 12 months significantly correlated with PFS. The estimated 60-month PFS rate was $87.6 \%$ with MCR and $40.4 \%$ without MCR by 12 months $(\mathrm{P}=0.0280)$ (Figure 6). Cytogenetic response by 12 months also significantly correlated with overall survival. The estimated 60 -month overall survival rate was $97 \%$ for patients with MCR and 47.4\% for those who did not achieve MCR by 12 months $(\mathrm{P}=0.0007)$.

Analysis of survival according to Sokal risk score was not significant, as shown in Figure $7(\mathrm{P}=0.1189)$. 


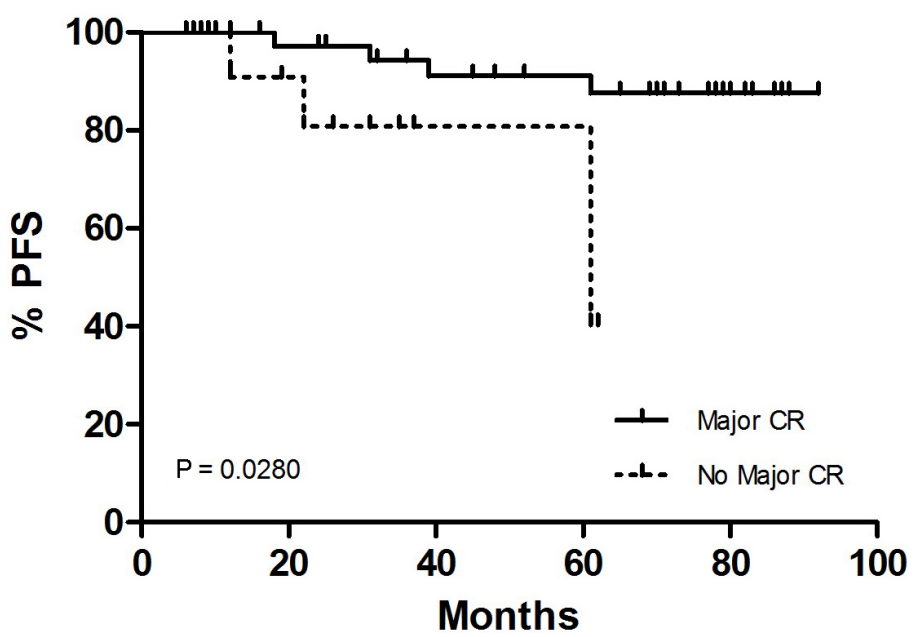

Figure 6. Progression-free survival (PFS) according to cytogenetic response (CR) by 12 months.

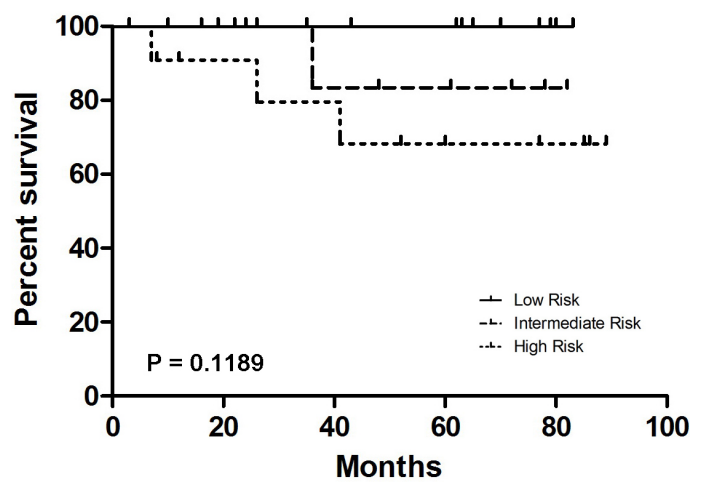

Figure 7. Overall survival according to Sokal risk score.

\section{Events}

The rates of events occurred as depicted in Table 2. The 60-month event-free survival rate was $65.5 \%$.

Table 2. Events considered to be adverse outcomes.

\begin{tabular}{lcc}
\hline Event & No. $(\%)$ & Time in months (median/range) \\
\hline Death & $6(8.6)$ & $26.5(7-41)$ \\
Progression & $12(17.1)$ & $35(3-71)$ \\
Accelerated phase & $10(14.3)$ & $18(16-18)$ \\
Blast crisis & $2(2.9)$ & $23(8-63)$ \\
Loss of response & $10(14.3)$ & $23(12-60)$ \\
Hematologic & $5(8.9)$ & 42 \\
Cytogenetic & $1(2.7)$ & \\
Molecular & & \\
\hline
\end{tabular}




\section{Treatment and toxicity}

At the end of the assessment, 40 patients (57.1\%) were still on the drug, while it had been discontinued in $30(42.9 \%)$. The main reasons for discontinuation were: $17(56.6 \%)$ failure or disease progression, $6(20 \%)$ death, $3(10 \%)$ loss of follow-up, $2(6.7 \%)$ patient decision, and 2 (6.7\%) toxicity. Thirty-three patients needed drug dose adjustment: eleven (15.7\%) required dose reduction; $6(8.6 \%)$ increase to $600 \mathrm{mg} ; 4(5.7 \%)$ increase to $800 \mathrm{mg}$, and 12 $(17.1 \%)$ increase to $600 \mathrm{mg}$ initially and then to $800 \mathrm{mg}$. Eleven patients $(15.7 \%)$ developed hematologic toxicity grades 3 or 4 .

\section{DISCUSSION}

Imatinib mesylate was initially introduced into clinical practice in patients resistant or intolerant to INF- $\alpha$ (Kantarjian et al., 2002a,b; Cervantes et al., 2003; Goldman, 2007). Since then, several publications have shown the results of treatment in similar groups (Kantarjian et al., 2004a,b; Funke et al., 2005; Lahaye et al., 2005). In this study, we analyzed patients at our institution who were treated with second-line IM and called them "pre-treated", as some were in late chronic phase (over a year since diagnosis) and others not. The treatment achieved similar results compared to those of some groups (Cervantes et al., 2003; Palandri et al., 2008) and better than others (Hocchaus et al., 2008), with reasonable tolerability and without observed adverse effects of greater severity.

The clinical characteristics of our group at diagnosis showed the presence of some variables related to worse prognosis. The proportion of patients with spleen size larger than 10 $\mathrm{cm}$ below the costal margin was around $21 \%$, while this has been observed by others at lower rates; the median number of leukocytes was higher when compared with other studies (Kantarjian et al., 2002a,b). These two features are clearly indicative of large tumor mass. Also, the prognostic stratification according to the Sokal risk score (Sokal et al., 1984) showed a smaller proportion of patients in the low-risk group (46\%), when compared to groups of high risk and intermediate risk, analyzed together (54\%). In other studies, however, there was a larger number of patients classified as low risk (Cervantes et al., 2003; Kantarjian et al., 2004a). Some reasons for these worse features may include delay in seeking medical help or no regular screening tests in many hospitals of the country. In spite of the unfavorable factors described, the good results observed could be explained by the lower median age of our group, with probably less associated diseases and possibly better compliance, since in younger patients we could see less severe or more tolerable adverse events.

Response rates to imatinib therapy in this long-term follow-up population were high and durable. Overall, $73.6 \%$ of patients achieved a major cytogenetic response, which was complete in $66.3 \%$. Thirty-four ( $85 \%$ ) patients still on treatment had achieved CCR, a substantial rate for late chronic-phase patients. These results are similar to some international studies (Kantarjian et al., 2004b). However, the continuous trend in improving responses was not seen in our group, which showed a stabilization rate around 48 months (Figure 1). This was probably seen because of the fewer number of cytogenetic analyses performed after achievement of CCR and start of molecular monitoring. Long-term outcomes were satisfactory. The estimated 5-year progression-free survival $(84.2 \%)$ and overall survival $(89.1 \%)$ were close to that observed in many series (Cervantes et al., 2003; Kantarjian et al., 2002b, 2004a,b; Lahaye et 
al., 2005), which may be due to reasonable cytogenetic control at the beginning of treatment.

We showed that the degree of cytogenetic response at 12 months was significantly associated with progression-free survival (Figure 6) according to findings by Hochhaus et al. (2008). However, it was not possible to show significant differences according to Sokal risk score in either major cytogenetic response $(\mathrm{P}=0.4898)$ (Figure 3$)$ or overall survival $(\mathrm{P}=$ 0.1189) (Figure 7). One possible explanation is the small number of patients in which the score was available (50\% of the entire group), or the fact that data of general population groups could not be equivalent to those observed in clinical trials, as observed by Lucas et al. (2008). Our data were insufficient to evaluate a difference in treatment outcomes with imatinib-intolerant patients and those who had failed treatment with interferon.

In regard to safety, the patients exhibited lower rates of hematologic toxicity $(15.7 \%$ grades 3 and 4) in relation to a phase 2 study that evaluated IM (Kantarjian et al. 2002a), in which up to $35 \%$ of patients showed grades 3 and 4 neutropenia. There were no reports of severe non-hematologic toxicity, and adverse effects were handled according to usual recommendations.

We share the opinion of Palandri et al. (2008) that the interest in the fate of the patients treated in late chronic phase tended to vanish over time. However, we also agree with the view that our patients and many living in other countries still in treatment may benefit from observations made in several centers, with respect to rates of recurrence or progression to more advanced forms of the disease or even the long-term stability of their responses.

In conclusion, treatment with imatinib mesylate of previously treated chronic-phase $\mathrm{Ph}$-positive CML patients induced durable responses in a high proportion of patients, despite producing adverse clinical and laboratory features at presentation. Sokal risk score was not applicable in our patients as a tool for stratification.

\section{ACKNOWLEDGMENTS}

We thank the staff from the Núcleo de Hematologia e Hemoterapia do Hospital de Base do Distrito Federal, Brasília, Brazil, for clinical collaboration, and Novartis Pharma Brazil, for support in cytogenetic and molecular studies of our patients.

\section{REFERENCES}

Baccarani M, Saglio G, Goldman J, Hochhaus A, et al. (2006). Evolving concepts in the management of chronic myeloid leukemia: recommendations from an expert panel on behalf of the European LeukemiaNet. Blood 108: 1809-1820.

Baccarani M, Cortes J, Pane F, Niederwieser D, et al. (2009). Chronic myeloid leukemia: an update of concepts and management recommendations of European LeukemiaNet. J. Clin. Oncol. 27: 6041-6051.

Bortolheiro TC and Chiattone CS (2008). Leucemia mielóide crônica: história natural e classificação. Rev. Bras. Hematol. Hemoter. 30: 3-6.

Cervantes F, Hernandez-Boluda JC, Steegmann JL, Conde E, et al. (2003). Imatinib mesylate therapy of chronic phase chronic myeloid leukemia resistant or intolerant to interferon: results and prognostic factors for response and progression-free survival in 150 patients. Haematologica 88: 1117-1122.

Cortes J and Kantarjian H (2003). Advanced-phase chronic myeloid leukemia. Semin. Hematol. 40: 79-86.

Druker BJ, Tamura S, Buchdunger E and Ohno S (1996). Effects of a selective inhibitor of the ABL tyrosine kinase on the growth of BCR-ABL1-positive cells. Nat Med. 2: 561-566.

Druker BJ, Talpaz MDM, Resta DJ, Peng B, et al. (2001). Efficacy and safety of a specific inhibitor of the BCR-ABL tyrosine kinase in chronic myeloid leukemia. New Engl. J. Med. 344: 1031-1037.

Funke VAM, Medeiro CR, Lima DH, Setúbal DC, et al. (2005). Therapy of chronic myeloid leukemia with imatinib 
mesylate in Brazil: a study of 98 cases. Rev. Bras. Hematol. Hemoter. 27: 159-165.

Giles FJ, Cortes JE, Kantarjian HM and O'Brien SM (2004). Accelerated and blastic phases of chronic myelogenous leukemia. Hematol. Oncol. Clin. North Am. 18: 753-74, xii.

Goldman JM (2007). How I treat chronic myeloid leukemia in the imatinib era. Blood 110: 2828-2837.

Goldman JM and Melo JV (2003). Chronic myeloid leukemia - advances in biology and new approaches to treatment. $N$. Engl. J. Med. 349: 1451-1464.

Hochhaus A, Druker B, Sawyers C, Guilhot F, et al. (2008). Favorable long-term follow-up results over 66 years for response, survival, and safety with imatinib mesylate therapy in chronic-phase chronic myeloid leukemia after failure of interferon- $\alpha$ treatment. Blood 111: 1039-1043.

Hughes TP, Kaeda J, Branford S, Rudzki Z, et al. (2003). Frequency of major molecular responses to imatinib or interferon alfa plus cytarabine in newly diagnosed chronic myeloid leukemia. N. Engl. J. Med. 349: 1423-1432.

Kantarjian HM, Sawyers C, Hochhaus A, Guilhot F, et al. (2002a). Hematologic and cytogenetic responses to imatinib mesylate in chronic myelogenous leukemia. N. Engl. J. Med. 346: 645-652.

Kantarjian HM, Talpaz M, O'Brien S, Smith TL, et al. (2002b). Imatinib mesylate for Philadelphia chromosome-positive, chronic-phase myeloid leukemia after failure of interferon-alpha: follow-up results. Clin. Cancer Res. 8: 2177-2187.

Kantarjian HM, Cortes JE, O'Brien S, Luthra R, et al. (2004a). Long-term survival benefit and improved complete cytogenetic and molecular response rates with imatinib mesylate in Philadelphia chromosome-positive chronic-phase chronic myeloid leukemia after failure of interferon-alpha. Blood 104: 1979-1988.

Kantarjian HM, O'Brien S, Cortes J and Giles F (2004b). Survival advantage with imatinib mesylate therapy in chronicphase chronic myelogenous leukemia (CML-CP) after INF- $\alpha$ failure and in late CML-CP, comparison with historical controls. Clin. Cancer Res. 10: 68-75.

Lahaye T, Riehm B, Berger U, Paschka P, et al. (2005). Response and resistance in 300 patients with BCR-ABL-positive leukemias treated with imatinib in a single center: a 4.5-year follow-up. Cancer 103: 1659-1669.

Lucas CM, Wang L, Austin GM, Knight K, et al. (2008). A population study of imatinib in chronic myeloid leukaemia demonstrates lower efficacy than in clinical trials. Leukemia 22: 1963-1966.

O'Brien SG, Guilhot F, Larson RA, Gathmann I, et al. (2003). Imatinib compared with interferon and low-dose cytarabine for newly diagnosed chronic-phase chronic myeloid leukemia. N. Engl. J. Med. 348: 994-1004.

Palandri F, Iacobucci I, Martinelli G, Amabile M, et al. (2008). Long-term outcome of complete cytogenetic responders after imatinib $400 \mathrm{mg}$ in late chronic phase, philadelphia-positive chronic myeloid leukemia: the GIMEMA Working Party on CML. J. Clin. Oncol. 26: 106-111.

Quintás-Cardama A and Cortes J (2009). Molecular biology of bcr-abl1-positive chronic myeloid leukemia. Blood 113: 1619-1630.

Sawyers CL (1999). Chronic myeloid leukemia. N. Engl. J. Med. 340: 1330-1340.

Silver RT, Woolf SH, Hehlmann R, Appelbaum FR, et al. (1999). An evidence-based analysis of the effect of busulfan, hydroxyurea, interferon, and allogeneic bone marrow transplantation in treating the chronic phase of chronic myeloid leukemia: developed for the American Society of Hematology. Blood 94: 1517-1536.

Sokal JE, Cox EB, Baccarani M, Tura S, et al. (1984). Prognostic discrimination in "good-risk" chronic granulocytic leukemia. Blood 63: 789-799.

Thijsen S, Schuurhuis G, van Oostveen J and Ossenkoppele G (1999). Chronic myeloid leukemia from basics to bedside. Leukemia 13: 1646-1674.

Vardiman JW, Melo JV, Baccarani M and Thiele J (2008). Chronic Myelogenous Leukaemia, BCR-ABL1 Positive. In: WHO Classification of Tumors of Hematopoietic and Lymphoid Tissues (Swerdlow SH, Campo E, Harris NL, Jaffe ES, et al., eds.). IARC, Lyon, 32-37. 